\title{
Primeros pasos del aprendizaje móvil en Costa Rica: Uso de WhatsApp como medio de comunicación en el aula
}

\author{
First Steps of Mobile Learning in Costa Rica: Use of WhatsApp as a Means of Communication \\ in the Classroom
}

\section{Primeiros passos do aprendizado móvel na Costa Rica: usando o WhatsApp como meio de comunicação na sala de aula}

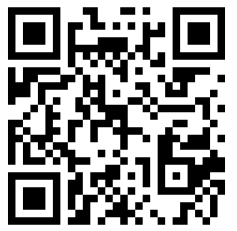

Jairo Guadamuz-Villalobos

Universidad de Costa Rica

San José, Costa Rica

jairo.guadamuz@ucr.ac.cr

iD http://orcid.org/0000-0002-0284-3656

Recibido • Received • Recebido: 19 / 09 / 2018

Corregido • Revised • Revisado: 20 / 02 / 2020

Aceptado • Accepted • Aprovado: 01 / 04 / 2020

\begin{abstract}
Resumen: Con el fin de conocer la opinión del estudiantado en relación con el uso de dispositivos móviles como apoyo al proceso de enseñanza y aprendizaje; y las posibilidades que existen de implementar aprendizaje móvil en el aula universitaria costarricense, la presente investigación documenta la integración de la herramienta Whatsapp como medio de comunicación en el aula universitaria, aplicada a los grupos 01, 02 y 03 del curso Referencia II, impartido en la Escuela de Bibliotecología y Ciencias de la Información de la Universidad de Costa Rica, durante el segundo ciclo del año 2017. Se muestra en detalle el proceso de implementación de la aplicación en el aula, la sistematización de los contactos, el uso de listas de difusión, el tipo de material enviado en las comunicaciones realizadas y la opinión del estudiantado que fue parte del proceso de investigación. Dicha valoración del estudiantado se analizó en relación con sus experiencias con otras herramientas de comunicación en el aula, su preferencia por utilizar de nuevo Whatsapp como herramienta de comunicación y su opinión general del ejercicio. Como resultado, se concluye que existen condiciones para realizar aprendizaje móvil en el aula universitaria costarricense, y su implementación trae beneficios en el proceso de enseñanza y aprendizaje, además, el estudiantado se mostró satisfecho con la experiencia. Finalmente se propone este ejercicio como un primer acercamiento de implementación de aprendizaje móvil en Costa Rica.
\end{abstract}

Palabras clave: Aprendizaje móvil; educación superior; enseñanza de la bibliotecología; tecnologías de información y la comunicación; Costa Rica. 
http://doi.org/10.15359/ree.24-2.18

ROR: https://ror.org/01t466c14 Universidad Nacional, Costa Rica

http://www.una.ac.cr/educare

educare@una.cr

Abstract: To have students' opinion on the use of mobile devices to support the teaching and learning process, and the existing possibilities of implementing mobile learning in the Costa Rican university classroom, this research documents the integration of the WhatsApp tool to communication in the university classroom. The research was conducted with groups 01, 02, and 03 of the Reference II course, taught in the School of Library and Information Sciences of the University of Costa Rica, during the second semester of 2017. It shows the process of implementation of the application in the classroom, the systematization of contacts, the use of broadcast lists, the material sent in the communications, and the students' opinions who were part of the research process. This assessment considered the relation between students and their experiences with other communication tools in the classroom, their preference for using WhatsApp again as a communication tool, and their general opinion on the exercise. In conclusion, there are conditions for mobile learning in the Costa Rican university classroom, and its implementation brings benefits in the teaching and learning process. Also, the students were satisfied with the experience. Finally, this exercise is proposed as the first approach to mobile learning implementation in Costa Rica.

Keywords: Mobile learning; higher education; teaching of librarianship; information and communication technology, Costa Rica.

Resumo: Esta pesquisa tem como objetivo conhecer a opinião dos alunos sobre o uso de dispositivos móveis para apoiar o processo de ensino e aprendizagem, e as possibilidades existentes para implementar a aprendizagem móvel na sala de aula da universidade da Costa Rica. A pesquisa documenta a integração da ferramenta WhatsApp como meio de comunicação na sala de aula da universidade, aplicada aos grupos 01, 02 e 03 do curso Referência II, ministrado na Escola de Biblioteconomia e Ciências da Informação da Universidade da Costa Rica, durante o segundo ciclo de 2017. O processo de implementação do aplicativo é demonstrado na sala de aula, a sistematização dos contatos, o uso da lista de divulgação, o tipo de material enviado nas comunicações realizadas e a opinião do grupo de estudantes que fizeram parte do processo de pesquisa. Essa avaliação dos alunos foi analisada em relação às suas experiências com outras ferramentas de comunicação em sala de aula, a preferência por usar o WhatsApp novamente como ferramenta de comunicação e a opinião geral do exercício. Como resultado, conclui-se que existem condições para a aprendizagem móvel na sala de aula da universidade da Costa Rica, e sua implementação traz benefícios no processo de ensino e aprendizagem; além disso, os alunos ficaram satisfeitos com a experiência. Finalmente, este exercício é proposto como uma primeira abordagem para a implementação do aprendizado móvel na Costa Rica.

Palavras chaves: Aprendizagem móvel; ensino superior; ensino de biblioteconomia; tecnologias de informação e comunicação; Costa Rica.

\section{Introducción}

La formación universitaria es la principal responsable de la preparación profesional de una nación, y la dinámica del aula debe, por tanto, responder a las necesidades actuales de aprendizaje de sus estudiantes $y$, a la vez, proponer el uso de herramientas que faciliten la investigación, el crecimiento académico y, por supuesto, la comunicación asertiva entre la persona docente y el estudiantado.

Jairo Guadamuz-Villalobos

Los artículos de la Revista Electrónica Educare del Centro de Investigación y Docencia en Educación de la Universidad Nacional, Costa Rica, se comparten bajo términos de la Licencia Creative Commons: Reconocimiento, № Comercial, Sin Obra Derivada 3.0 Costa Rica. Las autorizaciones adicionales a las aquí delimitadas se pueden obtener en el correo: educare@una.cr 
Por varios años, no solo en los escenarios de educación superior, se ha propuesto la integración de la tecnología para potencializar el trabajo en el aula. Según Astullido (2015), el uso de la tecnología en la educación personaliza el aprendizaje, fortalece el trabajo individualizado, mejora la atención a las opiniones del estudiantado, mejora la eficacia del proceso de enseñanza-aprendizaje, da sentido a lo aprendido y mejora la motivación, la interactividad y las posibilidades educativas.

Sin embargo, es importante no limitarse al uso de equipo de proyección de recursos multimediales y material de apoyo, la comunidad estudiantil sigue cambiando y, por lo tanto, estos esfuerzos ya no son suficientes, por lo que la integración de la tecnología en el aula debe cambiar también y, para ello, es importante promover, desde el ejercicio de la docencia, la búsqueda continua de nuevos recursos para apoyar el proceso de enseñanza aprendizaje y potencializar la experiencia del estudiante dentro y fuera del aula.

En síntesis, el uso de las tecnologías de información y comunicación (TIC) en el aula no solo trae consigo la agilización de actividades en el manejo de información, sino que además plantea un reto de constante evolución y desarrollo de las estrategias didácticas de acuerdo con las exigencias de las nuevas generaciones y las posibilidades ofrecidas por la tecnología. Así lo dejan ver Monsalve Castro y Monsalve Castro (2015) cuando afirman:

En la actualidad, la tecnología forma parte de la vida cotidiana y así mismo del contexto educativo. Para Salinas (1997), el sistema educativo requiere grandes cambios propiciados por la innovación tecnológica en los nuevos ambientes de aprendizaje. Esto implica nuevas concepciones en el proceso de enseñanza y aprendizaje, caracterizadas por la relación con el saber y las nuevas prácticas de docencia adaptadas al contexto actual. (p. 54)

Si las tecnologías están cambiando y las exigencias del estudiantado, y sus hábitos de consumo de información también, es necesario que dentro del aula el cambio también suceda. Surgen entonces las preguntas: ¿Hacia dónde nos llevan las nuevas tecnologías? ¿Qué nuevos dispositivos pueden llevarse al aula? ¿Qué nuevas estrategias pueden crearse con estos mismos?

Por lo tanto, con el fin de conocer la opinión del estudiantado en relación con el uso de dispositivos móviles como apoyo al proceso de enseñanza y aprendizaje; y las posibilidades que existen de implementar aprendizaje móvil en el aula universitaria costarricense, el presente artículo relata la experiencia de implementación de la aplicación WhatsApp como medio de comunicación entre el estudiantado y el profesor del curso Referencia II, impartido en la Escuela de Bibliotecología y Ciencias de la Información de la Universidad de Costa Rica. Dicha experiencia fue evaluada por el estudiantado mediante la aplicación de un breve cuestionario para conocer su opinión. Mediante la presentación de los resultados obtenidos en la evaluación del estudiantado y la crónica del desarrollo de implementación de la aplicación, se pretende sugerir esta práctica como una opción para integrar al aula el uso de dispositivos móviles como apoyo al proceso de enseñanza y aprendizaje. 


\section{Aprendizaje móvil}

La tecnología está modificando la educación tal cual la conocemos. Los últimos años han traído cambios relevantes a través del uso de internet y muy recientemente en el uso de tecnologías móviles. Khan et ál. (2015) señalan que el aprendizaje ya no está confinado al aula ni a personal instructor dentro de ella, ahora este proceso se desarrolla en nuevos horizontes donde, en cualquier lugar y en cualquier momento, puede ser aprovechado por cualquier persona que posea un dispositivo móvil. Esta nueva dinámica de aprendizaje se denomina Mobile Learning (M-Learning), o aprendizaje móvil en español, y ha traído consigo la apertura a nuevas experiencias dentro y fuera del aula. Así lo indica la Unesco (2013):

Estudiantes y profesores utilizan ya tecnologías móviles en diversos contextos para una extensa gama de finalidades docentes y de aprendizaje, y actores clave del ámbito educativo, desde los ministerios nacionales de educación hasta los distritos escolares locales, ensayan políticas de apoyo para impulsar el aprendizaje móvil e innovador en entornos educativos formales e informales. (p. 12)

Por su parte, Vidal Ledo et ál. (2015) definen aprendizaje móvil como "una metodología de enseñanza y aprendizaje valiéndose del uso de los teléfonos u otros dispositivos móviles, como son las agendas electrónicas y las tabletas o tablets, entre otros, con conectividad a Internet" (p. 670). Es decir, se trata del uso de dispositivos móviles en el aula; estos pueden ir desde la construcción compleja de diseños en realidad aumentada, hasta el simple uso de juegos lúdicos en los dispositivos del estudiantado. Esta gama de posibilidades incluye, por supuesto, el uso de dispositivos como medios de comunicación en el aula, mediante uso de aplicaciones como WhatsApp, Skype y Hangout, entre otras.

En todo el mundo el fenómeno está haciéndose presente. Así lo dejan claro Moreira, Ferreira et ál. (2017), quienes afirman que parte de las experiencias ofrecidas mediante el uso de dispositivos móviles en la educación, tanto en primaria como en secundaria, permiten el trabajo colaborativo entre estudiantes, el acceso a contenido digital y la posibilidad de aprendizaje personalizado. Por lo que el uso de dispositivos móviles en el aula ha dejado de considerarse una amenaza para pasar a convertirse en un recurso de apoyo al fortalecimiento del proceso de enseñanza-aprendizaje, tanto dentro como fuera de esta.

Una de las razones para intentar el aprendizaje móvil, es decir, integrar los dispositivos móviles al aula, es la cantidad de usos que pueden gestionarse con ellos. Se trata no solo de permitir que la comunidad estudiantil lleve su celular o su tableta a clases, sino que se debe además disponer de actividades pensadas para su desarrollo con esta tecnología. Según Boude Figueredo y Barrero (2017), entre las posibilidades que ofrece el uso de dispositivos móviles en el aula pueden enumerarse: 
Enriquecer la práctica docente, Relación de los contenidos teóricos con la realidad, Desarrollo deestrategias que no dependen de la conectividad, Apoyo a salidas pedagógicas, Aprendizaje entre pares, Formación de una segunda lengua, Desarrollo de competencias en el desarrollo de la información, Desarrollo de materiales educativos digitales de apoyo, Orientar el trabajo independiente, Desarrollo de prácticas de laboratorio, Fortalecimiento de habilidades dentro y fuera del aula, Apoyo al proceso de realimentación, Apoyo al proceso de evaluación, Desarrollo de procesos interdisciplinares, Fomentar procesos en donde el estudiante es el centro del proceso. (p. 133)

Jiménez Porta y Diez-Martínez Day (2018), por su parte, realizan una investigación en la que analizan los beneficios de utilizar aplicaciones y juegos como apoyo al proceso de enseñanza de lectura inicial. Analizan once aplicaciones, identifican sus características más sobresalientes y logran determinar cuáles de ellas favorecen actividades como la lectura de letras aisladas, sílabas, frases, entre otras. Su investigación llega incluso a identificar cuáles de estas once aplicaciones pueden apoyar procesos de aprendizaje relacionados a principios del alfabeto, conciencia fonológica y fonética, estructura del lenguaje y conductas de lectura. Entre las conclusiones encontradas en la investigación destaca:

La presencia constante del texto en la mayoría de las aplicaciones, a pesar de la multimodalidad que puede ser empleada en las apps y los videojuegos, nos lleva a remarcar la importancia del uso de la lectoescritura para acceder a la información. (Jiménez Porta y Diez-Martínez Day, 2018, p. 84)

La bibliografía consultada enlista también lugares, como Oriente Medio, Portugal y la Península Ibérica, donde ya ha sido posible la integración de celulares y tabletas como tecnología para la educación. En Costa Rica, según el Programa de la Sociedad de la Información y Comunicación (PROSIC) (2017) de la Universidad de Costa Rica, por cada 100 habitantes existen 143,8 suscripciones a líneas móviles, es decir, la cantidad de líneas conectadas a las redes móviles del país supera la cantidad de habitantes. Por otro lado, mientras que el 39,2\% de costarricenses tienen servicio de internet fijo, el 70,8\% cuenta con acceso a la red mediante dispositivos móviles. Por lo tanto, según los datos del PROSIC, puede afirmarse que existen posibilidades reales para la implementación de aprendizaje móvil en las aulas costarricenses.

Conociendo este panorama, es oportuno elegir un escenario óptimo para realizar un primer acercamiento a las experiencias de aprendizaje móvil implementadas en otros países. La comunicación en el aula, siendo esta la base donde descansa una experiencia de aprendizaje más asertiva, puede considerarse una de las primeras actividades a potencializar mediante el uso de tecnologías móviles. Considerando las implicaciones legales referentes al uso del celular por parte de estudiantes menores de edad, se determina que el aula universitaria es el escenario óptimo para la realización de este primer acercamiento. 
Por otro lado, según datos del $7 m o$ Estudio de Redes Sociales en Centroamérica y el Caribe, $79,80 \%$ de los sujetos entrevistados afirma que WhatsApp es su red social favorita, colocándola en segundo, detrás de Facebook con un 81,30\% (Melgar, 2018). Esta aplicación, WhatsApp, a diferencia de Facebook, ofrece una mayor penetración en cuanto a envío y recepción de mensajes se refiere, pues la dinámica del App consiste en la comunicación personal mediante el uso de conversaciones de chat, la creación de grupos y el uso de listas de difusión, mientras que Facebook basa su contenido en la publicación de información en el denominado muro, el cual, según los hábitos de consumo del público usuario, la información posteada podría no ser mostrada al público meta en su totalidad, debido al algoritmo utilizado en dicha red social, el cual se basa en mostrar contenido según la cantidad de clics que haya ejecutado el sujeto lector.

Según Farucci (2018), los últimos cambios realizados al algoritmo de Facebook incluyen "mostrar más publicaciones de familiares y amigos a los usuarios, y menos de páginas corporativas" (párr. 2). Tomando en cuenta que el uso de Facebook para fines académicos incluye la creación de páginas o grupos, estos tendrían menos visibilidad para el público usuario en comparación con las publicaciones de amistades y familiares. Es decir, si se envía el mismo mensaje por Facebook y por WhatsApp, debido a las condiciones de penetración de ambas aplicaciones, es más probable que el mensaje sea recibido y leído de forma exitosa en WhatsApp que en Facebook.

Existen iniciativas para integrar el uso de la aplicación Whatsapp al aula. Una de ellas es la implementada por Bouhnik y Deshen (2014), dos investigadores de la Bar-llan University, en Israel, que decidieron documentar el uso de grupos de Whatsapp para la comunicación en el aula. Según Bouhnik y Deshen (2014), WhatsApp permite la comunicación de cualquier persona que posea un teléfono inteligente, tenga una conexión a internet activa y haya instalado la aplicación. Esta cualidad de la aplicación le permite apropiarse de una de las características principales de las tecnologías móviles: la penetración.

Tomando en cuenta estas referencias teóricas y prácticas, la presente investigación eligió para su desarrollo el uso de Whatsapp como herramienta para la búsqueda de una primera aproximación de aprendizaje móvil en el aula de educación superior costarricense. Sin embargo, a diferencia de Bouhnik y Deshen, no se utilizó la opción de creación de grupos, pues el objetivo de la investigación consistía en establecer comunicación unidireccional entre cada estudiante y su profesor, de forma tal que la interacción interpersonal de los grupos de Whatsapp no era oportuna. Además, la privacidad de las consultas en estos espacios se compromete al mostrar todos los mensajes de igual manera a la totalidad de miembros del grupo. Este y otros motivos por los cuales se prescindió del uso de grupos se explicarán con detalle más adelante.

A continuación se narran las acciones realizadas para recolectar los números de teléfonos de los sujetos usuarios, inscribirlos en la agenda de contacto del teléfono utilizado por el profesor, los pormenores sobre la elección y envío de contenidos y la opinión del estudiantado ante dicha actividad.

6

Jairo Guadamuz-Villalobos

Los artículos de la Revista Electrónica Educare del Centro de Investigación y Docencia en Educación de la Universidad Nacional, Costa Rica, se comparten bajo términos de la Licencia Creative Commons: Reconocimiento, № Comercial, Sin Obra Derivada 3.0 Costa Rica. Las autorizaciones adicionales a las aquí delimitadas se pueden obtener en el correo: educare@una.cr 


\section{Inscripción de estudiantes y sistematización de contactos}

Tal como se mencionó, la implementación de esta investigación se realizó en los grupos 01, 02 y 03 del curso de Referencia II, impartido durante el segundo ciclo del año lectivo 2017 (del mes de agosto al mes de diciembre) en la Escuela de Bibliotecología y Ciencias de la información de la Universidad de Costa Rica. Los tres grupos, conformados por un total de 67 estudiantes, fueron impartidos por el autor del artículo.

WhatsApp es una aplicación que integra condiciones de red social, tales como el uso de una fotografía de perfil y la posibilidad de publicar estados visibles por los contactos agregados al teléfono. Con el fin de no exponer la vida personal del profesor a la comunidad de estudiantes inscritos en los cursos, se tomó la decisión de utilizar un segundo chip de servicios telefónicos, ya que el dispositivo utilizado por el profesor en el curso fue el mismo utilizado en su vida personal. Esta decisión es de suma importancia, pues el mezclar la comunicación personal con el ejercicio de la profesión docente puede ocasionar la recepción de consultas fuera del horario establecido en clases y la pérdida de privacidad por la parte docente.

Para lograrlo, fue necesario contar con un celular que tuviera la posibilidad de utilizar dos cuentas de WhatsApp de forma simultánea, de forma que, desde el mismo dispositivo, el docente podía acceder su cuenta personal y, en una aplicación aparte, la cuenta con la que se comunicaba con el estudiantado. Para contar con dos copias de la aplicación WhatsApp en un solo dispositivo fue necesario instalar la aplicación Parallel Space, descargada de la página Google Play Store.

También se compró un chip prepago para colocar en el teléfono. Es importante mencionar que no todos los teléfonos cuentan con doble slot para chip de servicios móviles, por lo que -si se desea replicar este ejercicio- debe tenerse a disposición un teléfono con esta característica. Es posible también realizar el ejercicio con dos teléfonos separados, pero esto obligaría a cada docente a cargar dos dispositivos de manera constante. Finalmente, se creó una segunda cuenta Google para el celular del profesor, para que fuera la receptora de la información de contacto del estudiantado y así mantener separadas la agenda de contactos personal de la agenda que se construiría para el curso.

Una vez separados el WhatsApp y la cuenta Google personal del profesor de aquellos que se utilizarían en el curso, se procedió a agregar los datos personales del estudiantado al teléfono del docente. Este proceso trajo consigo dos situaciones a valorar. La primera de ellas era el conseguir los datos de todo el estudiantado de forma rápida y la segunda era transcribir la información recolectada a la cuenta Google creada para dichos efectos de forma sistemática.

Para facilitar la recolección de datos, se utilizó un formulario creado en Google Forms, el cual se facilitó al estudiantado el primer día de clases mediante la proyección del enlace al formulario en la pizarra. Se les facilitó un espacio de 5 a 10 minutos durante dicha sesión para 
http://doi.org/10.15359/ree.24-2.18

ROR: https://ror.org/01t466c14 Universidad Nacional, Costa Rica

http://www.una.ac.cr/educare

educare@una.cr

que ingresaran su información mediante su celular y se revisó en el mismo momento que el total de estudiantes presentes coincidiera con el total de formularios realizados. Los datos solicitados al estudiantado fueron: número de cédula, número de carné universitario, nombre completo, correo electrónico, número de teléfono y curso matriculado. Este último dato permitía identificar, si el estudiante estaba matriculado en el grupo 01, 02 o 03. Dicha diferenciación fue útil a la hora de enviar mensajes cuyo contenido tuviera información exclusiva para uno de los tres grupos

Google Forms permite la generación de una hoja de cálculo con los datos recolectados en sus formularios, la cual se descargó para ser preparado para la importación de la información contenida a la agenda de contactos de Google.

Al ser la agenda de contactos de Google una base de datos, es posible la importación y exportación de información en formato CSV, sin embargo, para que esta importación se realice con éxito, los encabezados de las columnas deben coincidir con las variables que utiliza Google para identificar a sus contactos. Esta correspondencia se representa en la Tabla 1

Tabla 1: Correspondencia de datos recolectados con formato de contactos de Google

\begin{tabular}{lll}
\hline Nombre del datos & \multicolumn{1}{c}{ Descripción } & Correspondencia en Google \\
\hline Número de cédula & Valor numérico de 9 dígitos, único para cada persona & - \\
Carné universitario & $\begin{array}{l}\text { Valor alfanumérico de } 6 \text { dígitos, único para cada estudiante } \\
\text { de universidad }\end{array}$ & First Name \\
Nombre completo & Nombres y apellidos del estudiantado & E-mail Address \\
Correo electrónico & Dirección de correo electrónico personal, o de uso habitual & Mobile Phone \\
Número de teléfono & Número de teléfono personal inscrito en WhatsApp & Last Name \\
\hline
\end{tabular}

Nota: Elaboración propia.

Por lo tanto, para completar la importación, en la hoja de cálculo descargada de Google Forms se cambiaron los valores de algunos de sus encabezados, de forma tal que la primera fila del documento pasó de contener sus valores originales: marca temporal, número de cédula, carné universitario, nombre completo, correo electrónico, número de teléfono y curso matriculado. Y se actualizaron los valores necesarios para ser importados a la cuenta Google: marca temporal, número de cédula, carné universitario, First Name, E-mail Adress, Mobile Phone y Last Name.

En la columna Last Name los valores coinciden con las opciones brindadas en el formulario: BI2004 - Referencia II - Grupo 01, BI2004 - Referencia II - Grupo 02 y BI2004 - Referencia II -Grupo 03. 
Para simplificar estos datos y que aparezcan al lado del nombre de cada estudiante se procedió a simplificarlos con los valores BI2004 - 001, B/2004 - 002 y B/2004 - 003 respectivamente. Dichos datos corresponden a las siglas de curso que la Universidad dispone para cada uno.

Una vez lista la hoja de cálculo, se guardó en formato CVS, y se importó desde el servicio de importación de contactos disponible en la plataforma de correo de Google. Este proceso creó una ficha de contacto por cada uno de los registros ingresados por el estudiantado al formulario, y de forma casi inmediata, son reconocidos por la aplicación WhatsApp duplicada en el celular del profesor, el cual había sido vinculado previamente a la cuenta Google.

Cabe mencionar que el valor marca temporal es generado automáticamente por el sistema, pero no es tomado en cuenta para efectos de esta investigación. Por su parte, los valores número de cédula y carné universitario, al no tener consistencia con las variables de Google son enviados al espacio de notas de la ficha de contacto creada en la importación. Finalmente, el valor First Name muestra el nombre ingresado por cada estudiante, E-mail Address muestra la dirección de correo electrónico, Mobile Phone muestra el número de teléfono, lo que además permite identificar sus datos desde WhatsApp, y Last Name muestra la sigla del curso matriculado.

\section{Envío de información y resolución de consultas}

WhatsApp ofrece dos maneras de enviar mensajes de forma masiva. La primera de ellas, y la más común, es mediante la creación de grupos, los cuales permiten la interacción simultánea de todos sus miembros, es decir, al enviar un mensaje por medio de un grupo este es recibido por la totalidad de sus integrantes y, si es contestado, la respuesta será recibida de igual manera. Dicha dinámica de comunicación no ofrece privacidad en caso de que el estudiantado quiera hacer consultas al profesor acerca de temas sensibles, además provoca la recepción de mensajes no deseados por el resto de miembros del grupo que no necesariamente tienen interés en la consulta planteada al profesor.

Sin embargo, WhatsApp también ofrece el envío de difusiones. Este servicio consiste en la posibilidad de enviar un mensaje a determinada cantidad de contactos; pero, si este mensaje es contestado, la respuesta es recibida únicamente por la persona que envió la difusión. Este sistema facilita la comunicación, personaliza las consultas y evita la recepción de mensajes no deseados. Al contar con dichas ventajas, el sistema de difusión fue el elegido para que el profesor pudiera enviar contenido de apoyo al curso mediante la apliacación WhatsApp.

Se crearon, por tanto, tres listas de difusión, una por cada grupo del curso, y se inscribieron en estas mismas a todo el estudiantado matriculado, momento en el cual fue de utilidad el espacio que identifica el grupo matriculado por cada estudiante. Una vez creadas las listas, el profesor solo enviaba un mensaje por cada difusión y de inmediato 67 estudiantes recibían 
http://doi.org/10.15359/ree.24-2.18

ROR: https://ror.org/01t466c14 Universidad Nacional, Costa Rica

http://www.una.ac.cr/educare

educare@una.cr

la información enviada. Además, si cualquiera de estas personas contestaba a la información enviada, su respuesta solo sería recibida por el profesor, permitiendo así una comunicación privada entre estudiante y docente.

El desarrollo del curso de Referencia II durante el período de realización de la presente investigación incluyó el envío de enlaces a las presentaciones proyectadas en clase y enlaces a material de apoyo al contenido estudiado. En la Figura 1 se muestran capturas de pantalla con el material didáctico que se envió a la comunidad estudiantil.

Figura 1: Captura de pantalla de material didáctico enviado

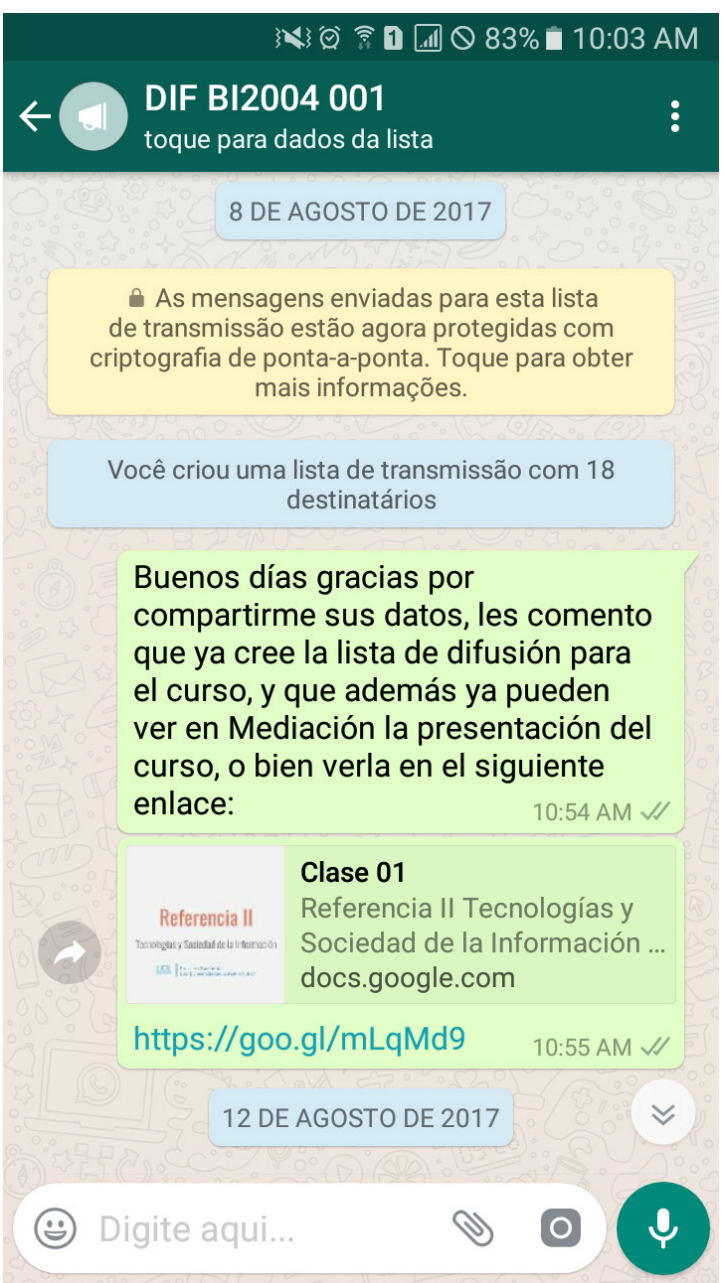

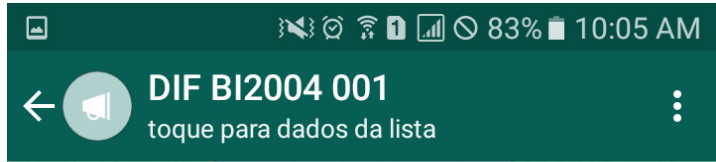

Hola, espero estén bien. Gravé un par de videos referentes al taller de la semana pasada, por lo que ambos pueden serles de mucha utilidad para la realización del examen. Les comento que en el video 2 , a partir del minuto 1:02:50 podrán ver el procedimiento para resolver el error de despliegue que estábamos teniendo con el Informe HTML de Zotero.

3:03 PM $\checkmark$

Taller de Diseño Web - Parte I

WiX

Clase virtual del curso

www.wix.com www.youtube.com

https://youtu.be/zcimDRkoaTA

3:03 PM $\checkmark$

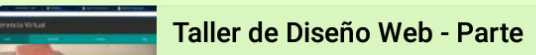

Clase virtual del curso Refer...

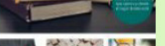

www.youtube.com

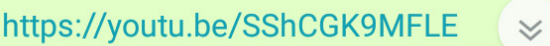
3:03 PM $\checkmark$

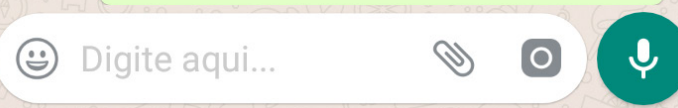

Nota: Whatsapp (Versión 2.18.217). Archivo personal. 
También se enviaron recordatorios de fechas importantes y la ubicación exacta de actividades realizadas fuera del aula. En la Figura 2 se muestra una captura de pantalla con las ubicaciones compartidas.

Figura 2: Captura de pantalla de ubicaciones compartidas

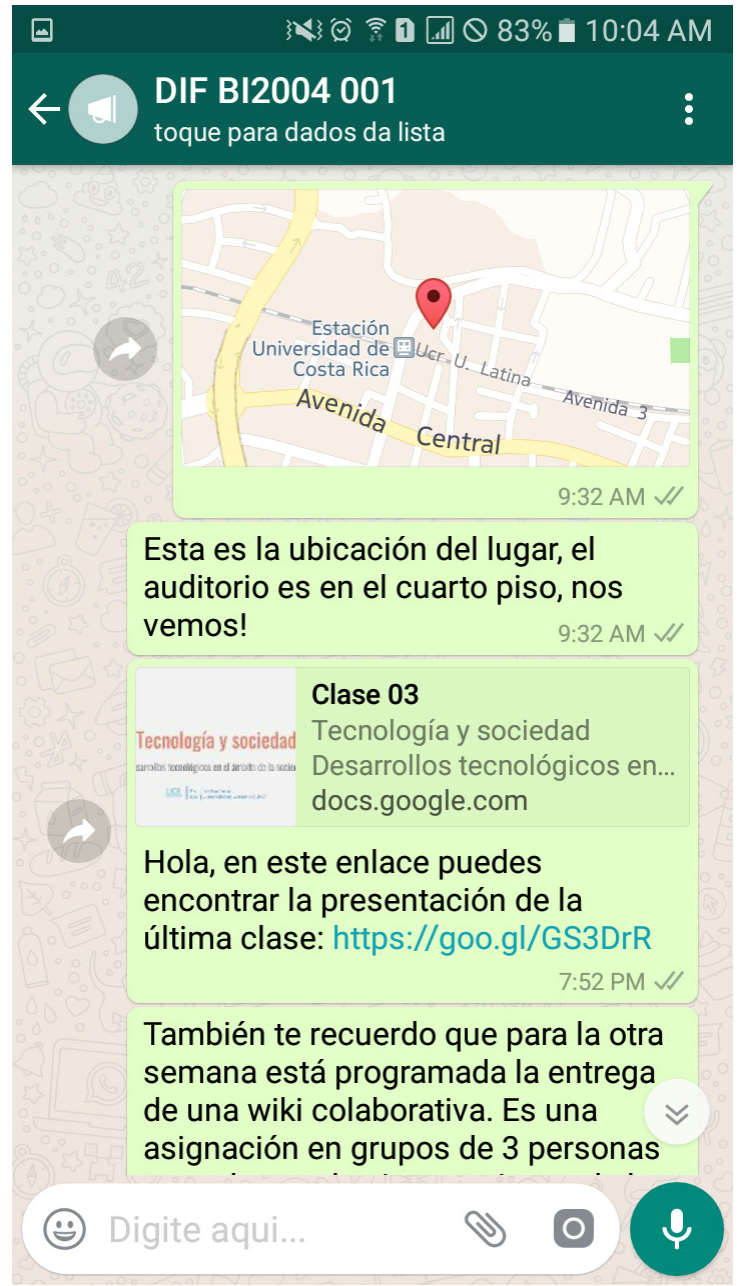

Nota: Whatsapp (Versión 2.18.217). Archivo personal.

Otra de las posibilidades a provechadas fue el envío de instrucciones de trabajos asignados y los temas de evaluación de exámenes y pruebas escritas. En la Figura 3 se muestran capturas de pantalla con las instrucciones, tareas y pruebas escritas enviadas a la comunidad estudiantil. 
http://doi.org/10.15359/ree.24-2.18

ROR: https://ror.org/01t466c14 Universidad Nacional, Costa Rica

http://www.una.ac.cr/educare

educare@una.cr

Figura 3: Captura de pantalla con envío de instrucciones, tareas y pruebas escritas
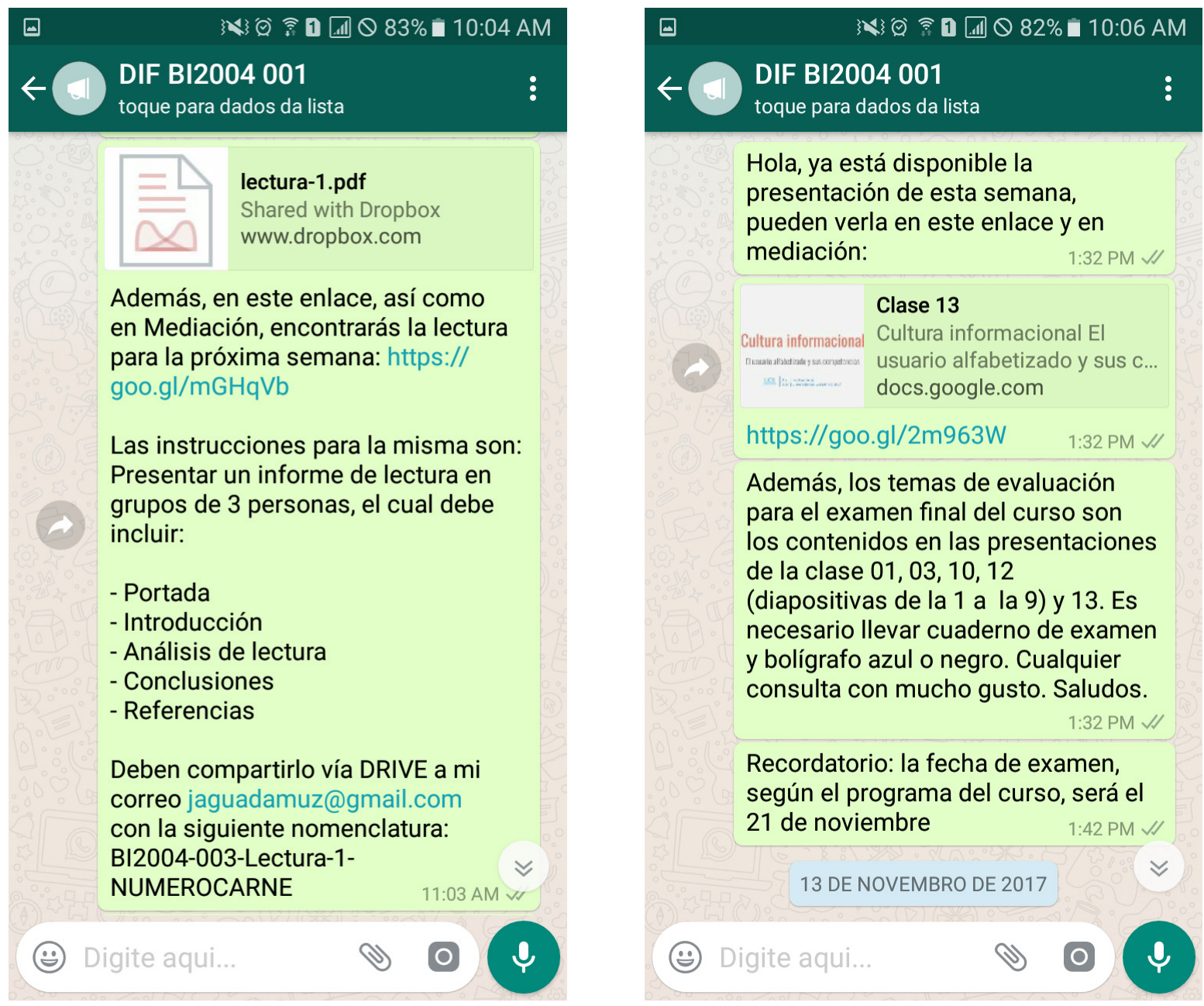

Nota: Whatsapp (Versión 2.18.217). Archivo personal.

Por su parte, el estudiantado podía realizar consultas individuales relacionadas al material enviado por el profesor o a algún otro tema referente al curso. En la Figura 4 se muestra una captura de pantalla de una de las consultas realizadas por la comunidad estudiantil. 
Figura 4: Captura de pantalla sobre realización de consultas

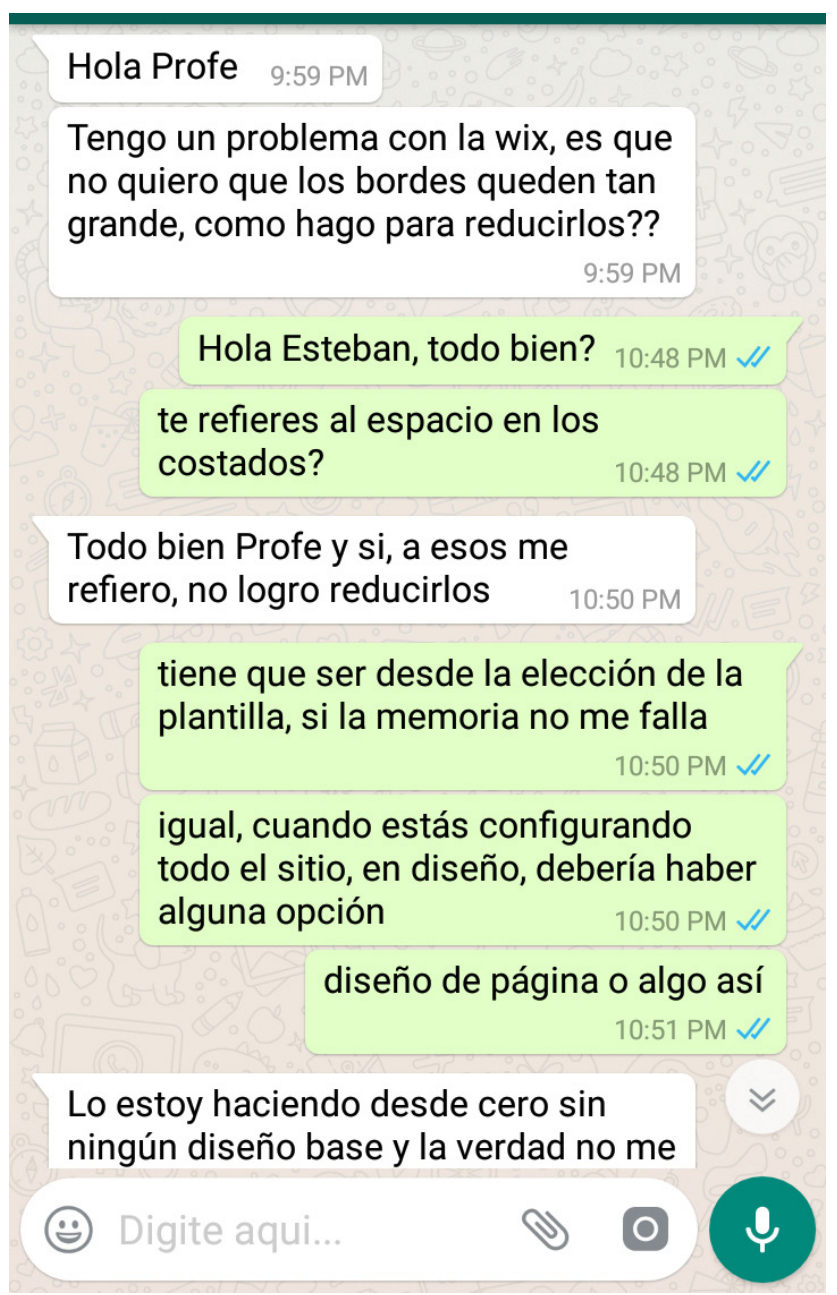

Nota: Whatsapp (Versión 2.18.217). Archivo personal.

Dichas consultas se realizaron aprovechando las capacidades multimediales de la aplicación, ya sea enviando fotografías, capturas de pantalla, archivos de audio o mensajes de texto.

\section{Opinión de la comunidad estudiantil}

Tras las 18 semanas de desarrollo del curso se consultó al estudiantado su opinión sobre la experiencia del uso de WhatsApp para recibir información general, la resolución de consultas y el envío de material didáctico de apoyo. Dicha información se recopiló mediante un breve 
censo aplicado a todas las 67 personas que formaron parte del proyecto. La selección de esta muestra se realizó por conveniencia, ya que el estudiantado participante estaba matriculado en tres grupos diferentes del mismo curso, impartidos por el investigador. A continuación, se detallan los resultados obtenidos.

La población de estudio se conformó por 49 mujeres, para un total de $73.1 \%$ y 18 hombres, con un $26.9 \%$. Es posible afirmar que se trató de una comunidad estudiantil joven, en su mayoría entre los 18 y 24 años, con un total de 59 personas, es decir un $88 \%$, y tan solo 8 entre los 25 y 35 años, para un $12 \%$ de la población estudiada.

La población en estudio ya había tenido experiencias en el uso de herramientas tecnológicas para la comunicación en clase, incluso algunas de las personas encuestadas afirman haber utilizado WhatsApp en otros cursos. En la Figura 5 se grafica la cantidad de respuestas con respecto a la utilización en otros cursos de otras herramientas tecnológicas para comunicarse con el docente.

Figura 5: Cantidad de estudiantes que han utilizado otras herramientas tecnológicas.

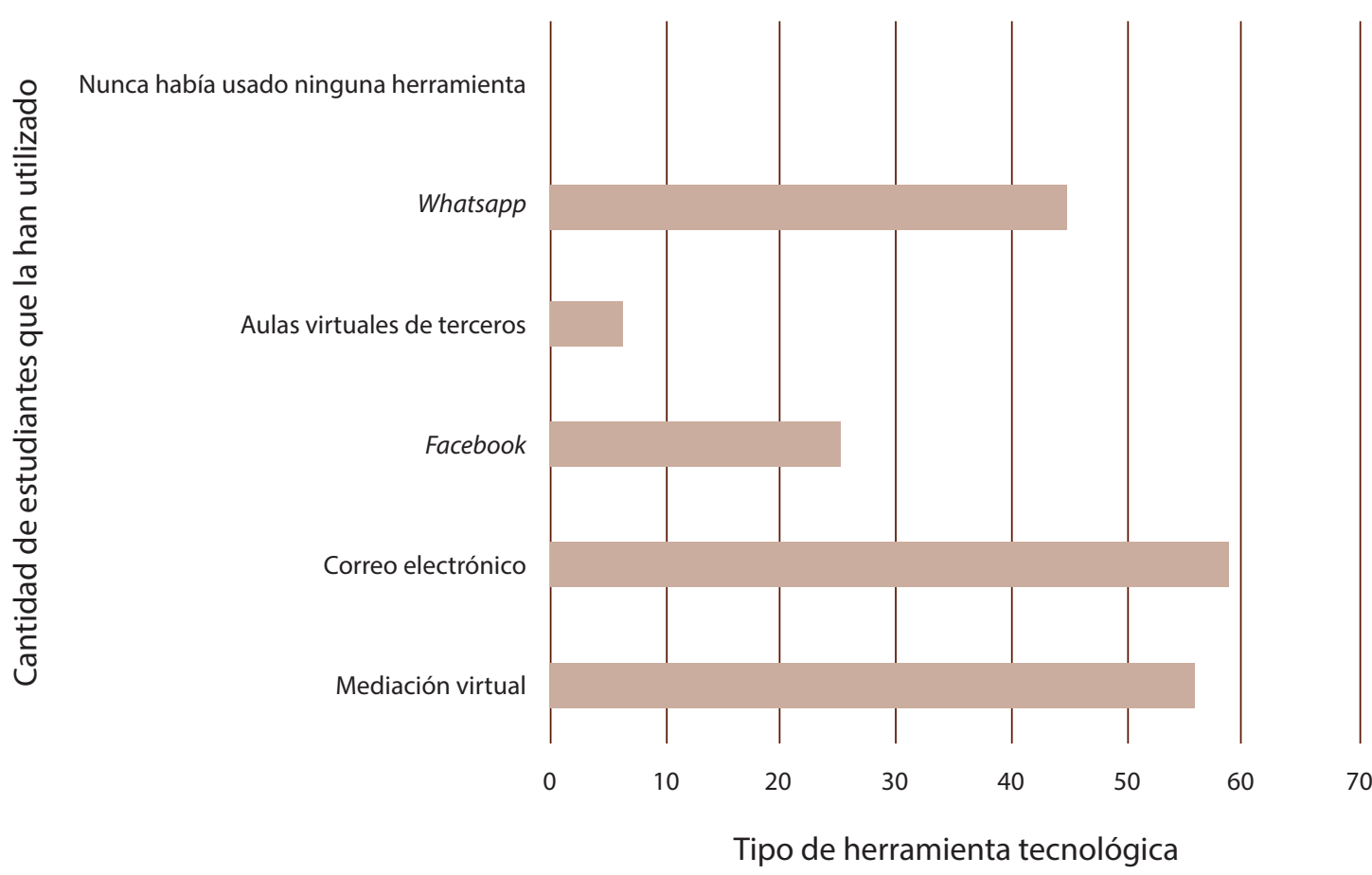

Nota: Elaboración propia. 
En su mayoría, el estudiantado ha utilizado herramientas como el correo electrónico y la plataforma de aulas virtuales y recursos compartidos de mediación virtual facilitada por la Universidad de Costa Rica, sin embargo, tras el ejercicio desarrollado durante el semestre, la mayoría del estudiantado coincide en preferir WhatsApp para comunicarse en el aula, con un 91\% de preferencia, lo cual supera de forma indiscutible las demás herramientas utilizadas anteriormente por el estudiantado. Es decir, aun teniendo experiencia en otras herramientas, el estudiantado inclina su preferencia en el uso de Whatsapp para comunicarse con el profesor, a pesar de que esta aplicación no ofrece funciones tan complejas como mediación virtual, las aulas virtuales de terceros o Facebook, sin embargo, en comparación con estas, sí ofrece inmediatez y portabilidad, características que podrían tomarse en cuenta como parte de las preferencias de comunicación que están teniendo las nuevas generaciones. En la Figura 6 se grafica el porcentaje de preferencia del estudiantado por el uso en el aula de herramientas como: mediación virtual, correo electrónico, Facebook, aulas virtuales de terceros y Whatsapp:

Figura 6. Herramientas de comunicación con el docente preferidas por el estudiantado.

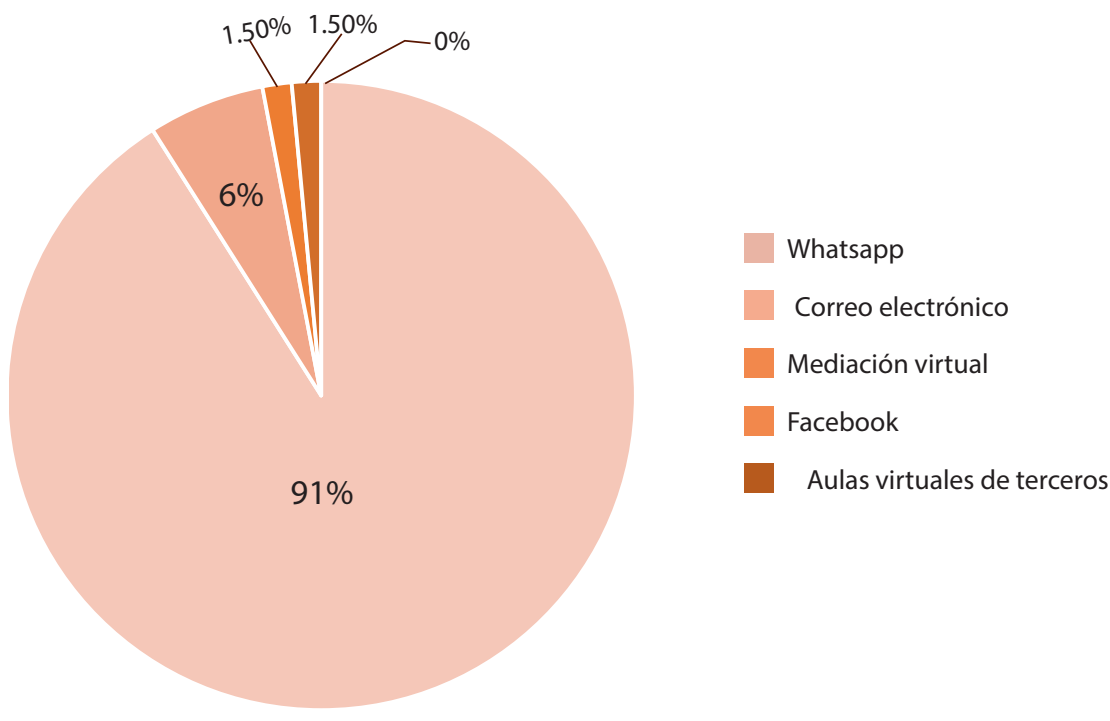

Nota: Elaboración propia.

Además, el 98.5\% consideró útil el uso de la aplicación como herramienta de comunicación entre estudiantes y profesor.

También se les consultó, de forma específica, cómo calificarían del 1 al 10 la utilidad de WhatsApp en cada uno de los tipos de contenidos enviados, es decir, avisos y recordatorios, material didáctico y resolución de consultas; donde 1 es nada útil y 10 es muy útil. Ante dichas preguntas se obtuvieron los siguientes resultados: 
http://doi.org/10.15359/ree.24-2.18

ROR: https://ror.org/01t466c14 Universidad Nacional, Costa Rica

http://www.una.ac.cr/educare

educare@una.cr

En el caso del envío de avisos y recordatorios 52 estudiantes calificaron de 10 el nivel de utilidad del uso de la aplicación, 11 la calificaron de 9, y 3 de ellos de 8 . Es decir, para enviar mensajes que recuerden fechas importantes, contenidos de evaluaciones o ubicaciones de eventos la mayoría de las personas encuestadas coinciden en que el uso de WhatsApp es útil para cumplir con estos procesos. De hecho, entre los comentarios adicionales del estudiantado destaca: Este medio es muy útil, ya que es algo que la mayoría de estudiantes utilizada todos los días constantemente (Pregunta 11, respuesta 35). Dicha aseveración coincide con la premisa anterior en la que se afirma que las nuevas generaciones se inclinan por contar con herramientas de comunicación inmediata y portable. Esta puede considerarse una primera práctica para avanzar hacia el aprendizaje móvil en el aula universitaria costarricense.

Por su parte, ante la evaluación de utilidad con respecto al envío de material didáctico de apoyo al curso, 43 estudiantes otorgaron la calificación más alta, 12 asignaron un 9 y 8 respondieron que tiene un nivel 8 de utilidad y finalmente 4 afirmaron que, en escala del 1 al 10, un 7 describe el nivel de utilidad del uso de la aplicación. De forma interesante, en el apartado de comentarios adicionales se puede encontrar la siguiente opinión: Excelente herramienta para llevar en orden y estar al corriente con el curso. (Pregunta 11, respuesta 11). Al ser Whatsapp una aplicación de mensajería instantánea que ordena los mensajes enviados y recibidos de forma cronológica, permite al estudiantado contar con una crónica de todas las comunicaciones realizadas en el ciclo al final del curso. Esta característica depende, por supuesto, del orden en que ejecute la comunicación la persona docente.

Con respecto a la resolución de consultas, 51 estudiantes dieron calificación máxima al uso de la aplicación, 8 afirmaron que merece un 9, 6 prefirieron calificarlo de 8, 1 lo calificó de 7 $y$, distante de la mayoría, tan solo 1 estudiante consideró que la calificación que merece el uso de la aplicación es de 5 . Entre los comentarios agregados por las personas encuestadas destaca el siguiente referido a la resolución de consultas: Eficiente; en especial a la hora de hacer consultas sobre algún trabajo, es más factible una respuesta rápida del profesor para poder continuar a tener que detener el avance hasta venir a la Universidad y evacuar las dudas (Pregunta 11, respuesta 40). En este sentido, es importante destacar que al usar Whatsapp como medio de comunicación con personas jóvenes se está creando una experiencia de interacción acorde con sus hábitos cotidianos, por lo que se acortan distancias entre el estudiantado y la persona docente.

No obstante, en virtud de comprender por qué una persona califica con tan solo 5 el nivel de utilidad de la aplicación, se toma en cuenta la una opinión expresada en el apartado destinado a comentarios adicionales: el único inconveniente es que en ocasiones las consultas por medio de mensajes es difícil de entender (Pregunta 11, respuesta 44). Este detalle evidencia que debe cuidarse la redacción de respuestas en todo momento, y que el uso de audios podría generar un mejor resultado en la resolución de consultas. Es importante utilizar todas las opciones que ofrece la aplicación para generar una comunicación más fluida y clara para todo el grupo participante.

16 Jairo Guadamuz-Villalobos

Los artículos de la Revista Electrónica Educare del Centro de Investigación y Docencia en Educación de la Universidad Nacional, Costa Rica, se comparten bajo términos de la Licencia Creative Commons: Reconocimiento, № Comercial, Sin Obra Derivada 3.0 Costa Rica. Las autorizaciones adicionales a las aquí delimitadas se pueden obtener en el correo: educare@una.cr 
Por otro lado, el estudiantado calificó, en su mayoría, con 10 el nivel de utilidad del uso de Whatsapp en el aula, con un total de 46 opiniones, 17 consideraron que la calificación más oportuna es de 9 , y tan solo 3 y 1 estudiante calificaron de 8 y 7 , respectivamente, el nivel de utilidad de la aplicación en términos generales. De hecho, una de las opiniones expresadas afirma que:

Me parece que el uso de la herramienta de Whatsapp ahora [sic] el tramite engorroso de enviar correos electrónico, hace la comunicación mas directa y fácil. En lo personal a veces me es difícil comunicarme con el profesor o profesora porque no se si les llego el correo, si este fue entregado o si quedo enterrado en una lista de correos spam que le llegan al docente. Muy bueno, en especial por lo práctico y cómodo que es recibir presentaciones, recordatorios, aclarar dudas, por que incluye no solo texto sino imagen y por sobre todo audio que facilita inimaginable mente el entendimiento de una explicación a distancia. (Pregunta 11, respuesta 43)

\section{Otra de las personas encuestadas también comentó:}

Me parece que fue una excelente opción el utilizar esta aplicación ya que se siente una atención mas personal por lo que me pareció muy buena la dinámica y me gustaría que mas personas implementaran el uso de esta herramienta. (Pregunta 11, respuesta 15)

Puede inferirse, entonces, que el uso de Whatsapp permite una atención más personalizada y acorde con los hábitos de comunicación del estudiantado, ofrece una experiencia más cercana entre estudiantes y docentes, mejora la comunicación y reduce brechas geográficas o temporales que otras herramientas pueden provocar.

Finalmente, el $100 \%$ de las personas encuestadas respondió que recomendaría el uso de WhatsApp como medio de comunicación en el aula a otros profesores y profesoras.

\section{Conclusiones y recomendaciones}

En una comunidad donde gran parte de sus miembros tiene acceso a dispositivos móviles, las aplicaciones de estos, en vez de representar una distracción, pueden convertirse en valiosas herramientas de apoyo a la comunicación en el aula y, por lo tanto, un refuerzo al proceso de enseñanza y aprendizaje. La comunicación es de vital importancia en la enseñanza, y el acompañamiento que el profesorado pueda dar desde afuera de las instalaciones educativas significa un valor agregado en la búsqueda de nuevos conocimientos por parte del estudiantado.

La tecnología ya ha aportado otras herramientas para la comunicación entre estudiantes y docentes; sin embargo, ninguna de ellas ofrece la versatilidad y la penetración que trae consigo el uso de WhatsApp. Aun cuando las plataformas de aprendizaje en línea ofrecen interfaces visibles desde un dispositivo móvil, las notificaciones, la facilidad de mantener la sesión siempre abierta y la rapidez de acceso a contactos hacen del WhatsApp una herramienta útil para comunicación en el aula. 
Adicionalmente, la aplicación ofrece distintas opciones multimedia que dan mejores posibilidades de acompañamiento al estudiantado sin importar la distancia. El envío de archivos adjuntos y enlaces de interés, la posibilidad de compartir imágenes o capturas de pantalla y la versatilidad que ofrecen las grabaciones de audio para compartir explicaciones más asertivas constituyen una gama de opciones de gran utilidad para intercambiar ideas, material didáctico y recursos relacionados al curso que se imparte.

Por otro lado, las nuevas generaciones tienen una mejor respuesta a los procesos de comunicación, si estos suceden en los medios y aplicaciones que usan en su cotidianidad. Trasladar la comunicación del aula al lenguaje y ambiente que el estudiantado domina facilita la interacción, reduce las brechas comunicativas provocadas por barreras generacionales o diferencias académicas e incentiva al estudiantado a mantenerse en contacto, no solo con su docente, sino también con sus pares estudiantes y con la materia vista en clase, lo cual trae consecuencias positivas en los procesos de enseñanza y aprendizaje.

No obstante, como todo medio de comunicación, es importante cuidar del mensaje, el canal y el lenguaje que se utilizan. La redacción a la hora de explicar debe ser clara, cuidar los signos de puntuación y la ortografía, pues en comunicación escrita cualquier uso equivocado de estas tres características puede cambiar el significado de una expresión. También es recomendable apoyarse en los recursos multimedia (imágenes, audio y video) para facilitar la comunicación.

El uso de tecnología en el aula no incluye solamente la proyección de material de apoyo durante la clase. Es posible encontrar muchas más posibilidades de integración tecnológica a través de dispositivos móviles, aplicaciones colaborativas, actividades virtuales y muchas otras opciones más. El personal docente debe sumarse a la tarea de buscar constantemente nuevas herramientas que faciliten el proceso de enseñanza y aprendizaje, de forma que, en un esfuerzo integrado, consiga mejorar la experiencia en el aula y acercar a sus estudiantes a las nuevas tecnologías de información y comunicación.

Finalmente, las nuevas prácticas de aprendizaje móvil son aplicables en aquellas comunidades estudiantiles dondeel alumnadotengaacceso no soloa dispositivos móviles sino también a conexiones de red que faciliten el uso de esta tecnología. Utilizar los dispositivos móviles en la educación permite generar una extensión del aula que mejora el acompañamiento docente a estudiante.

\section{Declaración de material complementario:}

El preprint del artículo está disponible en: https://doi.org/10.5281/zenodo.3351784

\section{Referencias}

Astullido, M. (2015). La integración de las tecnologías de la información y la comunicación en el diseño y la puesta en práctica de estrategias de enseñanza en las ingenierías en el contexto educativo superior mexicano [Tesis doctoral]. Universidad de Costa Rica, San José, Costa Rica.

18

Jairo Guadamuz-Villalobos

Los artículos de la Revista Electrónica Educare del Centro de Investigación y Docencia en Educación de la Universidad Nacional, Costa Rica, se comparten bajo términos de la Licencia Creative Commons: Reconocimiento, No Comercial, Sin Obra Derivada 3.0 Costa Rica. Las autorizaciones adicionales a las aquí delimitadas se pueden obtener en el correo: educare@una.cr 
Boude Figueredo, O. R. y Barrero, I. (2017). Diseño de estrategias de aprendizaje móvil a través de ambientes mezclados de aprendizaje. Sophia, 13(2), 96-105. https://dx.doi.org/10.18634/ sophiaj.13v.2i.572

Bouhnik, D. y Deshen, M. (2014). WhatsApp goes to school: Mobile instant messaging between teachers and students. Journal of Information Technology Education: Research, 13, 217-231. https://doi.org/10.28945/2051

Farucci, C. (24 de enero de 2018). Cambios de algoritmo de Facebook: Últimas modificaciones [Artículo de blog]. https://carlofarucci.com/cambios-de-algoritmo-de-facebook2018\#EdgeRank realiza uno de los mayores cambios en el algoritmo de Facebook peligra el alcance enero 2018

Jiménez Porta, A. M. y Diez-Martínez Day, E. (2018). Análisis del contenido de apps y videojuegos: Implicaciones en procesos cognitivos en la lectura inicial. Apertura, 10(1), 71-87. https:// doi.org/10.32870/Ap.v10n1.1114

Khan, A. I., Al-Shihi, H., Al-khanjari, Z. A. y Sarrab, M. (2015). Mobile Learning (M-Learning) adoption in the Middle East: Lessons learned from edicationally advanced countries. Telematics and Informatics, 32(4), 909-920. https://doi.org/10.1016/j.tele.2015.04.005

Melgar,J.(30 deenerode2018).7moestudioderedessocialesdeCentroaméricayel Caribe2018[Blog]. Ilifebelt. https://ilifebelt.com/estudio-de-redes-sociales-de-centroamerica/2018/01/

Monsalve Castro, N. Y. y Monsalve Castro, C. (2015). La inclusión de la computadora en el aula por docentes de quinto grado de básica primaria como herramienta para propiciar el aprendizaje significativo en los estudiantes. Revista Escuela de Administración de Negocios, 79, 50-63. https://doi.org/10.21158/01208160.n79.2015.1267

Moreira, F., Ferreira, M. J., Pereira Santos, C. y Durão, N. (2017). Evolution and use of mobile devices in higher education: A case study in Portuguese Higher Education Institutions between 2009/2010 and 2014/2015. Telematics and Informatics, 34(6), 838-852. https:// doi.org/10.1016/j.tele.2016.08.010

Programa Sociedad de la Información y el Conocimiento (PROSIC). (2017). Informes Hacia la sociedad de la información y conocimiento 2017. Universidad de Costa Rica, PROSIC. http:// www.prosic.ucr.ac.cr/informe-2017-0

Unesco. (2013). El futuro del aprendizaje móvil: Implicaciones para la planificación y la formulación de políticas. Autor. http://unesdoc.unesco.org/images/0021/002196/219637s.pdf

Vidal Ledo, M. J., Gavilondo Mariño, X., Rodríguez Díaz, A. y Cuéllar Rojas, A. (2015). Aprendizaje móvil.Educación Médica Superior, 29(3), 669-679.http://scielo.sld.cu/scielo.php?script=sci arttext\&pid=S0864-21412015000300024\&lng=es\&tIng=es 